\title{
Numerical Investigation of Aerodynamic Characteristics of Wing of Ichoku-18 Unmanned Aerial Vehicle
}

\section{Abubakar Isah ( D Dankade@hotmail.com )}

Air Force Institute of Technology https://orcid.org/0000-0002-8998-0417

Mohammed Shariff Lawal

Air Force Institute of Technology

Ibrahim Yahuza

Air Force Institute of Technology

Abdulmajid Na'inna

Air Force Institute of Technology

\section{Research Article}

Keywords: Turbulence model, Drag and Lift coefficients, Aerodynamic efficiency and Angle of attack

Posted Date: January 18th, 2022

DOI: https://doi.org/10.21203/rs.3.rs-1194453/v1

License: (c) (i) This work is licensed under a Creative Commons Attribution 4.0 International License. Read Full License 


\section{Abstract}

An Unmanned Aerial Vehicle (UAV) whose wing consist of a combination of two different airfoils presents opportunity for improve aerodynamic performance; and research in this area has potential for significant innovation. This study employed a Computational Fluid Dynamic (CFD) technique to predict drag and lift coefficients and aerodynamic efficiency of the wing of Ichoku-18 (IU-18) UAV. The wing of the IU-18 UAV consists of two different aerofoil sections, NACA2415 at the root and SD7032 at the tip. The study investigates the aerodynamic characteristics of the wing at different Angle of Attack ( $A \circ A), A \circ A=0^{\circ}, 4^{\circ}$, $8^{\circ}, 12^{\circ}, 16^{\circ}, 20^{\circ}$ and $24^{\circ}$. Using a standard and realizable $k-\varepsilon$ turbulence models, the minimum and maximum drag coefficients and aerodynamic efficiencies were obtained at $A \circ A=0^{\circ}$ and $A \circ A=24^{\circ}$. Both turbulence models predicted the critical angle of attack occurs at $A \circ A=16^{\circ}$. The results obtained were in agreement with analytical design data of the UAV and findings from the literature. While the standard k- $\varepsilon$ turbulence model overpredicted the drag coefficients of the wing of IU-18 UAV, the values predicted by the realizable $k-\varepsilon$ turbulence model were in closer agreement with the experimental data. Also, the lift coefficients predicted by the realizable $k-\varepsilon$ turbulence model were more realistic and in closer agreement with that obtained analytically. Similarly, while the realizable $k-\varepsilon$ turbulence models prediction of the CLmax is in closer agreement with the analytical data, the standard $k-\varepsilon$ model underpredicted the variation of aerodynamic efficiency with AoA.

\subsection{Introduction}

The use of unmanned aerial vehicles (UAVs) in many aspects of human life, from local and industrial to military applications, is rapidly increasing. The various applications of UAVs have triggered the interest of scientists and engineers globally to advance the state of the technology. Advancing UAVs technology requires extensive design data that ensures the performance characteristics continuously fulfil the mission's purpose. The wing geometry has a significant influence on the performance characteristics of any aircraft, and this depends on the aerofoil section. The aerofoil is the building block of the the wing that produces most of the forces required to sustain all flight conditions [1-3]. As such, the design characteristics of the wing geometry is crucial. Hence, it is important to accurately predict the wing aerodynamic characteristics of any UAV, as it significantly affects the aircraft flight performance.

Due to the influence of the aerofoil sections on the wing aerodynamic performance, various types can be found in a wing, from root to tip [4, 5]. In addition, taper, twist and sweepback are added to the wing geometry to increase its efficiency. In addition to the wing, the generation of aerodynamic forces of drag and lift to sustain flight conditions also depends on the flowfield properties and the angle of attack (AoA). The AoA is the angle between a reference line on an aircraft (often the chord line of an aerofoil) and the approaching airflow [6]. It has been shown that AoA has a dominant effect in determining the optimum profile of a wing as compared to the airspeed [7]. Therefore, the aerodynamic analysis of a UAV at different AoA is vital for optimizing the wing geometry and flight performance. 
The numerical method of computational fluid dynamics (CFD) is widely employed for aerodynamic analysis to optimize initial design calculations at lesser cost and time. Furthermore, CFD provides significantly added information, such as the effect of three-dimensional vortices, which cannot be easily obtained experimentally $[1,8]$. In addition to the high cost of wind tunnel testing, they may also suffer from scaling effects. Consequently, CFD approach has become a veritable design tool significantly employed in accelerating the design and development of modern aircraft.

The Air Force Research and Development Center (AFRDC) in collaboration with the Air Force Institute of Technology (AFIT) has successfully designed the lchoku-18 (IU-18) UAV in the year 2016. The UAV is planned for large-scale manufacturing by the year 2025. However, the wing of the IU-18 UAV was developed analytically based on design requirements without wind tunnel testing or CFD analysis required to optimize its aerodynamic characteristics. Therefore, this study aims to employ the CFD approach to predict the aerodynamic characteristics of IU-18 UAV. The paper is structured as follows. It starts with a review of the related literature, followed by a description of the numerical method employed. Subsequently, results obtained are presented and analysed to derive the major conclusions from the study.

\subsection{Review Of Related Literature}

Numerous studies have investigated the aerodynamic performance of different kinds of aerofoils and wing configurations depending on the design and mission requirements using CFD and experimental techniques. A CFD study in [9] analysed the performance of winglets, blended and split scimitar winglets using $k-\omega$ shear stress transport (SST) turbulence model. The results demonstrated an increase in the lift generated by the wing and a decrease in the drag, thereby increasing the fuel mileage by up to $6-8 \%$ due to the blended winglet. Another study investigated aerodynamics of NACA0012 finite wing at low Reynold's numbers and $A o A=0{ }^{\circ}$ and $90^{\circ}$ using Spalart-Allmaras (S-A) turbulence model. The study found the turbulence model to be effective for wing simulation at low speeds. Also the study used the CType grid topology to generate a fine mesh around the wing. Eftekhari and Al-obaidi in [10] added that the C-Type grid topology is an efficient meshing method for wing geometry requiring fine mesh for accurate results. The study indicates the drag coefficient increases as the AoA is increases, resulting in a change in the flowfield from laminar to turbulent, with flow separation in between. The results revealed the lift coefficient linearly increases with AoA until it reaches its maximum value at $32^{\circ}$, which is the stall angle. As observed in the study, increasing the AoA beyond $32^{\circ}$ resulted in a decrease in the lift coefficient, reaching its minimum value at $A O A=90^{\circ}$. The results of this study were further validated experimentally with minor error of $3 \%$ at AoA less than $15^{\circ}$. However, at $A o A=20^{\circ}$, the percentage error obtained was $19 \%$. The larger error is due to flow separation at the leading wing edge. Refining the mesh around the leading edge reduced the percentage error obtained. The stall condition reported in the above study occurs as airflow becomes fully separated, when the AoA of the wing is above a critical value. 
The study found that changes in AoA greatly affects the aerodynamic characteristics of the aerofoil. Another study by Roy et al. in [11] experimentally and numerically investigated the effect of wing tip vortices on flow separation. The author employed the $k-\omega$ SST turbulence model in conjuction with steady-state solution to study unswept and untwisted NACA4415 rectangular wing at different AoA. Roy et al. in [11] further asserted the $k-\omega$ SST turbulence model predicted good agreement with experimental data for adverse pressure gradient and boundary-layer flows. To simulate the AoA, the directions of the stream velocity were adjusted according to the required AoA. The results of the study revealed that by increasing the AoA, the point of separation and the onset of stall moves near the leading edge. Shaha and Pachapuri in [12] studied and performed validation of NACA2415 aerofoil. They found that while AoA greatly affects aerodynamic characteristics, the coefficient of moment remains almost constant. Kandil and Elnady in [13] made similar conclusion. The study also revealed that in comparisons with other methods employed, the CFD results were in closer agreement with the experimental data.

An experimental and computational study carried out by Genç et al. in [14] examined the flow over NACA2415 aerofoil with and without a leading-edge slat at a transitional Reynolds number $\left(R e=2 \times 10^{5}\right)$. The experimental results shows AoA affect the aerodynamic coefficients of the aerofoil with critical value occuring at $A o A=12^{\circ}$ without the leading-edge slat and $C_{L \max }=1.3$. This results is approximately the same with that from the experimental study in [12]. However, as presented in [12], the theoretical data of NACA2415 shows that at $12^{\circ}$ AoA, the $C_{L}=1.5602$ while the CFD results predicted the $C_{L}=1.2103$. The computational technique in [14] employed four turbulence models using a C-type structured and unstructured grid on the aerofoil. Comparison of the numerical results with experimental data revealed, the $k-k_{L}-\omega$ turbulence model better predicted the lift curve as well as the stall. However, the same model did not provide good prediction of the reduction in separation buble associated with improved airflow due to the slat.While $k-\omega$ SST poorly predicted the lift curve, it better predicted the disappearance of the separation buble in agreement with the experiment. These inconsistencies in numerical results could be attributed to many factors, including but not limited to the type of turbulence models or discretization employed.

The literature reviewed shows, due to peculiarity of the wing of IU-18 UAV consisting of two different aerofoils, there is no CFD study related to its aerodynamic characteristics. Addressing this gap is the main motivation of this study. The study employed CFD technique to investigate the aerodynamic characteristics of the wing of Ichoku-18 UAV atAoA $=0^{\circ}, 4^{\circ}, 8^{\circ}, 12^{\circ}, 16^{\circ}, 20^{\circ}$ and $24^{\circ}$. The CAD model of the wing was developed using CATIA V5 as shown in Figures 1.

\subsection{Numerical Method}

The numerical method employed in this study entails solving the general governing equations of fluid flow, the Navier-Stokes equations (NSE). By solving the equations numerically for a specific set of boundary conditions, the flow properties in a given geometry are predicted $[2,15]$. In this study, the flowfield is apprximated as a steady, turbulent and viscous incompressible flow. Hence, the relevant form 
of the NSE is the Reynolds-average Navier-Stokes (RANS) equations, which are split into mean and fluctuating components [16]. The total velocity, $u_{i}$ is decomposed as a function of the mean velocity $u_{i}$ and the fluctuating component $u_{i}$ as shown in the Eq 1. In the same manner, averaging can be applied to all other flow variables as shown in Eq 2 for pressure.

$$
u_{i}=\bar{u}_{i}+u_{i}
$$

1

$$
p=\bar{p}+\stackrel{\square}{p}
$$

2

The averaged from of the continuity and momentum equations are given in Eq 3 as:

$$
\frac{\partial}{\partial X_{i}}\left(\bar{u}_{i}\right)=0
$$

$$
\frac{\overline{D u_{i}}}{D t}=-\frac{1}{\rho} \frac{\partial P}{\partial x_{i}}+\frac{\partial}{\partial x_{j}}\left[\mu\left(\frac{\partial \bar{u}_{i}}{\partial x_{j}}+\frac{\partial \bar{u}_{i}}{\partial x_{i}}-\frac{2}{3} \delta_{i j} \frac{\partial \bar{u}_{k}}{\partial x_{k}}\right)\right]+\frac{\partial}{\partial x_{j}}\left(\begin{array}{r}
-, \\
\left.-u_{i} u_{j}\right)
\end{array}\right)
$$

4

In The above equations, $u, \rho$ and $\mu$ represents the velocity, density and dynamic viscosity of the fluid. The

$$
,
$$

term $-u_{i} u_{j}$ in Equation 4 is known as the Reynolds stress tensor (RST) and is defined in Equation 5. The RST accounts for the turbulent fluctuations and is required to be modelled to closed the RANS equation. Modeling the RST to obtain a solution requires some approximations in the governing differential equations at various levels of sophistication, leading to different turbulence models employed in predicting engineering flows. However, each turbulence model has some limitations, depending on the nature of the flowfield [11]. Today, numerous commercial and proprietary software have been developed for CFD analysis of various industrial and non-industrial flows. In this study, ANSYS-Fluent 14.0 CFD software is employed in modeling the flowfield of IU-18 UAV with the aim of predicting complex interaction of lift and drag forces involved.

The coefficients of drag and lift force $C_{d}$ and $C_{l}$ are defined in Equations 6 and 7, respectively. 


$$
C_{d}=\frac{F_{d}}{0.5 \rho V^{2} A}
$$

$$
C_{l}=\frac{F_{l}}{0.5 \rho V^{2} A}
$$

7

where $F_{d}$ and $F_{l}$ are the drag and lift forces, respectively. $\rho$ is the density of the air, $A$ is the frontal area of the vehicle, and $V$ is the relative speed.

The true aerodynamic efficiency of the wing is defined by the ratio of $C_{l}$ and $C_{d}[4]$ as.

$$
\text { Aerodynamicefficiency }=\frac{F_{l}}{F_{d}}=\frac{C_{l}}{C_{d}}
$$

8

Determining the Reynolds number of the flowfield, $R e$ is vital in selecting the turbulence models in numerical analysis. $R e$ is a non-dimensional parameter that represents the ratio of inertia and viscous forces [17]. It provides a quantitative representation of the degree of turbulence in a flow and is represented in Equation $9[18,19]$.

$$
R e=\frac{\text { Inertiaforce }}{\text { Viscousforce }}=\frac{\rho V D}{\mu}
$$

9

where $\rho, V, D$ and $\mu$ is the air density, free stream velocity, characteristic length and dynamic viscosity, respectively. The $D$ for an aerofoil is equal to the aerofoil length. For a wing, $D$ is equal to the aerofoil chord length. While for a non-uniform wing, $D$ is equal to the Mean Aerodynamic Chord, MAC length [20]. For fully developed turbulent flow, $R_{e}>\approx 10^{6}$ [17].

For the IU-18 UAV at cruising altitude, the Reynolds number is obtained based on Equation 9 as follows:

$$
R e=\frac{0.771 \times 46 \times 0.9}{1.66 \times 10^{-5}}=1.923 \times 10^{6}
$$

Thus, the flow can be approximated as fully turbulent at the cruising altitude of the UAV. However, this does not eliminate the presence of low Reynolds number regions in the flowfield, particularly, at high AoA, where separation is highly likely.

Based on the Reynolds number of the IU-18 UAV computed above, the standard and realizable $k-\epsilon$ turbulence models are selected for this study. The standard $k-\epsilon$ model proposed by Launder and Spalding [21] is the most widely used turbulence model for industrial applications. The model has been 
acknowledged to be robust, economical and reasonably accurate for a wide range of industrial applications of fully developed turbulent flows. However, it performs poorly for flows with strong separation, large streamline curvature and large pressure gradient. Hence, in this study, the Realizable $k-\epsilon$ turbulence model will be employed for the purpose of comparison. This model offers the good performance among the versions of $k-\epsilon$ model. Hence, it is considered appropriate for modelling flows with separation and complex secondary flow feature $[22,23]$. The numerical computation of the flowfield investigated in this study was carried out using Ansys-Fluent 14.0 CFD code. The details of the computeraided design (CAD) of the wing geometry, numerical setup, boundary conditions and turbulence models employed are presented in the following subsections.

\subsection{CAD Model of Wing of IU-18 UAV}

The wing of the Ichoku-18 UAV has the following dimensions: a span of $11 \mathrm{~m}$, a root chord of $1.20 \mathrm{~m}$, a tip chord of $0.50 \mathrm{~m}$, an aspect ratio of 11.68 and a gross area of $10.36 \mathrm{~m}^{2}$, Table 1 presents the remaining specifications of IU-18 UAV wing.

Table 1

Specifications of Wing of Ichoku-18 UAV.

\begin{tabular}{|ll|}
\multicolumn{2}{|c|}{ Specifications of Wing of Ichoku-18 UAV. } \\
\hline Constraint & Dimensions \\
\hline Trailing edge sweep angle & $4.45^{\circ}$ \\
\hline Leading-edge sweep back angle & $4.39^{\circ}$ \\
\hline MAC & $0.90 \mathrm{~m}$ \\
\hline Fitness ratio & 13.0 \\
\hline Anhendral & $3^{\circ}$ \\
\hline Twist & $-2^{\circ}$ \\
\hline Taper ratio & 0.42 \\
\hline Leading-edge sweep & $0^{\circ}$ \\
\hline
\end{tabular}

\subsection{Numerical Setup}

\section{a) Geometry design modeller}

The CAD model of the wing developed in CATIA V 5 was situated in a computational domain (CD) with dimensions as shown in Figure 2. Subsequently, the CD was imported into ANSYS-Fluent 14.0 software for meshing and analysis. This study employed the C-type CD due to its simplicity and time savings [24]. 
Due to symmetric nature of the wing, only half of its CAD model was developed. To model variation in flight attitude, the study opted for changing the flow field velocity vectors and its orientation relative to the wing. This has been shown to present the same effect as changing the flow AoA as described in [24] and and employed by Roy et al. in [11].

\section{b) Boundary conditions}

The boundary conditions prescribed include 'Inlet Velocity' on the first face of the CD in the Z-direction, "Pressure Outlet" on the face opposite the inlet, and "wall" on the wing model under investigation. The rectangular walls of the CD were defined as 'Symmetry'. Figure 3 shows the boundaries conditions prescribed on the $\mathrm{CD}$.

\section{c) Meshing}

In this study, an unstructured mesh was generated on both the wing model and CD. To improve the grid quality, advance size function, relevance centre and smoothing in Ansys Fluent 14.0 were changed to 'Proximity and Curvature', 'Fine' and 'High', respectively. The Proximity and Curvature option was employed to refine the mesh over much of the model and control the mesh density in regions of the model where features are located more closely together without using numerous local controls. The fine smoothing option allows controlling the variation in the size of the mesh elements thereby improving the accuracy of the numerical analysis. The relevance centre provide finer mesh in the swept regions closer to the geometry [24]. Thereafter, the meshing is generated and done on both the wing model and CD.

To ensure the solution obtained is independent of the grid size, grid independence check was done before adopting the finer grid size. This was achieved through performing the simulation with finer grid size. The results of the check show that the analysis grid size has some effect on the CFD results. However, as show on Table 2, the effect of using the finer the grid on the values of $\mathrm{Cd}$ and $\mathrm{Cl}$ obtained was less than an order of magnitude, which is not significant. Figure 4 shows the mesh generated on the wing model and the CD. Subsequently, the finer mesh consisting of 86,546 nodes and 482,214 elements for the wing and the $C D$, respectively was adopted for the remaining calculations.

Table 2

Grid independence check results.

\begin{tabular}{|llllll|}
\hline Grid size & AoA & Nodes & Elements & Cd & Cl \\
\hline Coarse & $0^{\circ}$ & 67,654 & 376,935 & 0.044 & 0.321 \\
\hline Finer & $0^{\circ}$ & 86,546 & 482,214 & 0.026 & 0.246 \\
\hline
\end{tabular}

\section{d) Setup}

The model was set up using a three-dimensional, steady-state, pressure-based coupled algorithm. The turbulence model implemented in this study is the standard and realizable $k-\epsilon$ turbulence model. The input parameters are the cruising speed and altitude of the UAV, given as $46 \mathrm{~m} / \mathrm{s}$ and 4,572 m, respectively. The remaining input parameters relate to properties of the surrounding air at altitude of 
4,572 m, namely, pressure, viscosity, density and temperature, which are given as $57.182 \mathrm{kPa}$,

$1.66 \times 10^{-5} \mathrm{~kg} / \mathrm{m} . \mathrm{s}, 0.771 \mathrm{~kg} / \mathrm{m}^{3}$ and $258.43 \mathrm{~K}$, respectively.

\subsection{Solution initialization}

To initialize the solution from the boundary conditions specified, a "Hybrid Initialization" was employed. Hybrid initialization is a collection of recipes and boundary interpolation methods. It solves the Laplace equation to produce a velocity field that conforms to complex domain geometries, and a pressure field that smoothly connects high and low-pressure values in the CD. All other variables (velocity, pressure, turbulence and VOF) were patched based on domain averaged values or a predetermined recipe [25].After initialization, seven (7) different cases namely; $\mathrm{AoA}=0^{\circ}, 4^{\circ}, 8^{\circ}, 12^{\circ}, 16^{\circ}, 20^{\circ}$ and $24^{\circ}$ were simulated for the two turbulence models. Lift and drag coefficients values were monitored and saved for each iteration. The convergence history was also monitored. The residual Root-Mean-Square (RMS) Error default value was set to $10^{-6}$ for better convergence. In some simulation where convergence was not possible, calculations were run for a stabilized solution determined by low fluctuations in the residuals.

\subsection{Results And Discussion}

This section presents and discusses the results obtained from the study and the key findings. Figures 5 and 6 illustrate the results of $\mathrm{C}_{\mathrm{D}}$ and $\mathrm{C}_{\mathrm{L}}$ obtained from the simulation after the solution has been stabilized for $\mathrm{AoA}=00^{\circ}, 4^{\circ}, 8^{\circ}, 12^{\circ}, 16^{\circ}, 20^{\circ}$ and $24^{\circ}$.

It can be seen from Figure 5 that at $\mathrm{AoA}=0^{\circ}, 4^{\circ}, 8^{\circ}, 12^{\circ}, 16^{\circ}, 20^{\circ}$ and $24^{\circ}$, the $\mathrm{C}_{\mathrm{D}}$ obtained were respectively $0.026,0.043,0.068,0.099,0.132,0.164$ and 0.194 , for the standard $k-\epsilon$ turbulence model. The corresponding $C_{D}$ obtained were respectively $0.016,0.032,0.056,0.085,0.117,0.144$ and 0.170 for the realizable $k-\epsilon$ turbulence model. The constant increase in the drag coefficient with increasing AoA as predicted by both turbulence models is not surprising. This is because the increase in the drag is caused majorly by an increase in the pressure exerted on the wing, which is a result of an increase in the wing surface area exposed to the free stream. Also, due to the quest for greater lifting ability by increasing the AoA, lift induced drag is generated, adding to the total drag produced by the wing. It can be observed that the minimum drag coefficients occurs at $\mathrm{AoA}=0^{\circ}$; and the value is obtained to be 0.026 and 0.016 for the standard and realizable $k-\epsilon$ turbulence models respectively, see Figure 5 . However, the study of Hamza in [26] using Athena Vertex Lattice (AVL) method, obtained drag coefficient of 0.030 at $A O A=0^{\circ}$ for the wing of IU-18 UAV. Considering the latter study was carried out for wing and tail sections of the IU-18 UAV, the higher value of drag coefficient obtained is quite understandable. The presence of the tail section has the effect of increasing the $\mathrm{Cd}$. Thus, it can be deduced that the minimum drag coefficients predicted by both turbulence model are realistic.

The value of Cd obatained experimentally by Genç et al. in [14] for a wing with NACA2415 aerofoil at AoA $=0{ }^{\circ}$ was 0.01 . This also confirms that the value of $\mathrm{Cd}$ predicted by the realizable $k-\epsilon$ turbulence model is in closer agreement with the experimental data. Thus, the experimental result suggests the 
standard $k-\epsilon$ turbulence model overpredicted the drag coefficients of the wing of IU-18 UAV. The variations between the value of $C d$ obatained experimentally in [14] with that predicted by the realizable $k-\epsilon$ turbulence model reported in this study could be associated with differences in design of the wing of the IU-18 UAV, which has twists and two different airfoil sections. These translates into differences in drag coefficients. The maximum drag coefficient obtained at $A o A=24^{\circ}$ is 0.194 and 0.17 for the standard and realizable $k-\epsilon$ turbulence models respectively, see Figure 5 .

Figure 6 shows the curves of variation of lift coefficients with AoA for the two turbulence models. It can be easily seen in Figure 6 that an increase in the AoA causes increase in the lift coefficients for both the standard and realizable $k-\epsilon$ turbulence models. At AoA $=0^{\circ}, 4^{\circ}, 8^{\circ}, 12^{\circ}, 16^{\circ}, 20^{\circ}$ and $24^{\circ}$ the values of the lift coefficients obtained were respectively $0.246,0.339,0.419,0.479,0.512,0.509$ and 0.467 for the standard $k-\epsilon$ turbulence model. In the case of the realizable $k-\epsilon$ turbulence model, the corresponding lift coefficients are $0.264,0.369,0.461,0.536,0.581,0.569$ and 0.500 , see Figure 6 . At $A o A=0^{\circ}$, the minimum lift coefficients values of 0.246 and 0.264 were predicted by the standard and realizable $k-\epsilon$ turbulence models, respectively. However, as obtained in [26], at $A o A=0^{\circ}$ using AVL method, the lift coefficient of the IU-18 wing was found to be 0.377 . Considering the latter study was carried out for wing and tail sections of the IU-18 UAV, the higher value of lift coefficient obtained is quite understandable. Similarly, the analytical method of Jemitola and Abbe in [27] shows the lift coefficient of IU-18 UAV at $A o A=0^{\circ}$ is 0.389 . Thus, it can be deduced that the minimum lift coefficient of 0.264 predicted by the realizable $k-\epsilon$ turbulence model is more realistic and in closer agreement with that obtained analytically.

Figure 6 also shows that, above AoA $=16^{\circ}$, the lift coefficient generated stop increasing, and starts to decrease. This revealed that $A O A=16^{\circ}$ is the critical angle of attack of the wing of IU-18 UAV as predicted by both turbulence models. At this AoA, both the standard and realizable $k-\epsilon$ turbulence models predicted similar value of maximum lift coefficients $C_{L_{\max }}$ of 0.512 and 0.581 , respectively. $A$ $\mathrm{CL}_{\text {max }}$ value of 0.74 was obtained at AoA of $15.6^{\circ}$ from the analytical design of the IU-18 UAV by Jemitola and Abbe in [27]. It worthy of note that this higher value of $\mathrm{CL}_{\max }$ obtained analytically was for the whole UAV. Hence, it is evident that the values predicted by both turbulence models are realistic. Neverthless, realizable $k-\epsilon$ turbulence models prediction of the $\mathrm{CL}_{\max }$ is in closer agreement with the analytical data.

To prevent stalling at high AoA the wing requires an optimization of the aerofoil through incorporation of high lift devices. The effectiveness of such high device is seen in [14], where adding a leading-edge slat led to increase of $6^{\circ}$ in the stalling AoA, with a $50 \%$ increase in the $\mathrm{CL}_{\max }$. This rationalizes the need for the design modifications of the IU-18 UAV wing buy incorporating high lift devices.

The predicted variation of aerodynamic efficiencies of the wing at various AoA for the two turbulence models tested is presented in Figure 7 
Figure 7 shows the aerodynamic efficiencies predicted by the standard $k-\epsilon$ turbulence model at AoA $=0^{\circ}, 4^{\circ}, 8^{\circ}, 12^{\circ}, 16^{\circ}, 20^{\circ}$ and $24^{\circ}$ were $9.46,7.88,6.16,4.84,3.88,3.10$ and 2.41 respectively. Whereas, the realizable $k-\epsilon$ turbulence model predicted $16.50,11.53,8.23,6.31,4.97,3.95$ and 2.94 respectively. The results shows, the maximum aerodynamic efficiencies of the wing occurs at the least AoA, AoA $=00^{\circ}$, which have values of 9.46 and 16.5 as predicted by the standard and realizable $k-\epsilon$ turbulence models, respectively. On the other hand, the lowest aerodynamic efficiencies of the wing were obtained at the highest AoA, $A O A=24^{\circ}$ for both models. This revealed that the wing exhibited greater aerodynamic efficiency at $A o A=0^{\circ}$. Subsequently, there is a consistent decrease in the efficiency with increase in AoA. Thus, at $A o A=0^{\circ}$, we obtained the most lift for the least amount of drag generated. When the AoA is increased beyond $0^{\circ}$, it can be observed that the drag coefficient increased more than the lift coefficient. It has been observed that the increase in AoA further decreases the aerodynamic efficiency until the stalling angle is reached, and keeps decreasing even beyond the critical AoA.

Therefore, the smaller the AoA the greater the aerodynamic efficiency. Thus, it can be deduced that there is no economic advantage in cruising at $A o A>0^{\circ}$ because more fuel will be consumed, leading to more emission of pollutants to the environment, a great source of concern today.

Based on results presented in Figure 7, the standard $k-\epsilon$ model underpredicted the variation of aerodynamic efficiency with AoA, relative to the values obtained from the realizable $k-\epsilon$ model. These discrepancies are likely associated with significant separation and adverse pressure gradient present in the flowfield at higher AoA, for which the standard $k-\epsilon$ model has been reported to have poor performance [28].

\subsection{Conclusion}

This study employs CFD approach to investigate the aerodynamic performance of wing of IU-18 UAV at different angles of attack by considering its cruising phase of flight. Results of the analyses show that while the standard $k-\epsilon$ turbulence model overpredicted the drag coefficients of the wing of IU-18 UAV, the values predicted by the realizable $k-\epsilon$ turbulence model were in closer agreement with the experimental data. Overall, results obtained with the realizable $k-\epsilon$ turbulence model were more realistic in comparison with data obtained experimentally and analytically. Therefore, this research has made a substantial contribution to knowledge by modelling the aerodynamics performance of the wing of the IU-18 UAV. Also, the study was able to determine the relative performance of two RANS turbulence models in predicting the flowfield at different AoA.

\section{Declarations}

\section{Availablity of data and materials}

Not applicable

\section{Competing interest}


The authors declare that they have no competing interests to disclose.

\section{Funding}

Not applicable

\section{Authors contributions}

Al was the major contributor in carrying out the analysis as it is a part of his master's degree thesis. MSL was the supervisor of the masters degree research and made significant inputs in developing the manuscript. IY and AN contributed with ideas for interpretation of the results and review of the manuscript. All authors read and approved the final manuscript.

\section{Acknowledgements}

The authors would like to appreciate the Air Force Research and Development Center (AFRDC) and the Air Force Institute of Technology (AFIT) for providing lots of design input parameters for the analysis and validation.

\section{Author details}

Not applicable

\section{References}

1. Merryisha S, Rajendran P (2019) Experimental and CFD Analysis of Surface Modifiers on Aircraft Wing: A Review. CFD Lett 11:46-56

2. Narendiranath BT, Parmenshwar P, Beladiya A, Bhabhra A (2017) CFD Analysis and Comparison using Ansys and Star-CCM+ Of Model Aerofoil Selig 1223. Int J Mech Eng Technol 8:312-318

3. Yayli UC, Kimet C, Duru A et al (2017) Design optimization of a Fixed Wing Aircraft. Adv Aircr Spacecr Sci 4:65-80. https://doi.org/10.12989/aas.2017.4.1.065

4. Anderson JD (2011) Fundamentals of Aerodynamics, Sixth. McGraw-Hill Education, New York

5. Cakir M (2012) CFD Study on Aerodynamic Effects of a Rear Wing/Spoiler on a Passenger Vehicle. Santa Clara University, California

6. Hiremath S, Malipatil AS (2014) CFD Simulations of Aircraft Body with Different Angle of Attack and Velocity. Int J Innov Res Sci Eng Technol 3:16965-16972. https://doi.org/10.15680/IJIRSET.2014.0310077

7. Nigam PK, Tenguria N, Pradhan MK (2017) Analysis of horizontal axis wind turbine blade using CFD. Int J Eng Sci Technol 9:46-60. https://doi.org/10.4314/ijest.v9i2.5 1

8. Lawal MS, Thomas S, Udeagulu C, Jemitola PO (2015) Prediction of Aerodynamic Paramaters and Pressure Distribition of the Wing of Gulma UAV. In: 3rd International Symposium on Engineering and Natural Science (ISEAN). Beijing 
9. Arunkumar K, Chinthamani P, Nema D et al (2018) Performance Analysis of Winglet Using CFD. In: International Conference on Aeronautics, Astronautics and Aviation (ICAAA). pp 151-157

10. Eftekhari S, Al-obaidi ASM (2019) Investigation of a NACA0012 Finite Wing Aerodynamics at Low Reynold's Numbers and $0{ }^{\circ}$ to $90^{\circ}$ Angle of Attack. J Aerosp Technol Manag 11: e1519:1-11. https://doi.org/10.5028/jatm.v11.1023

11. Roy A, Mallik AK, Sarma TP (2018) A Study of Model Separation Flow Behavior at High Angles of Attack Aerodynamics. J Appl Comput Mech 4:318-330. https://doi.org/10.22055/JACM.2018.24339.1183

12. Shaha SN, Pachapuri MSA (2015) NACA 2415- Finding Lift Coefficient Using CFD, Theoretical and Javafoil. IJRET Int J Res Eng Technol 4:444-449

13. Kandil MAF, Elnady AO (2017) Performance of GOE-387 Airfoil Using CFD. Int J Aerosp Sci 5:1-7. https://doi.org/10.5923/j.aerospace.20170501.01

14. Genç MS, Kaynak Ü, Lock GD (2009) Flowover an aerofoil without and with a leading-edge slat at a transitional Reynolds number. Proc Inst Mech Eng Part G J Aerosp Eng 223:217-231. https://doi.org/10.1243/09544100JAER0434

15. Khuntia SK, Ahuja AS, Base N (2018) Optimal Design and CFD Analysis of Wing of a Small-Scale UAV to Obtain Maximum Efficiency. Int J Comput Appl 1:148-164. https://doi.org/10.26808/rs.ca.i8v1.15

16. Aftab SMA, Rafie ASM, Abdul Razak NA, Ahmad KA (2016) Turbulence Model Selection for Low Reynolds Number Flows. PLoS ONE 11:1-15. https://doi.org/10.1371/journal.pone.0153755

17. John JB, Russell MC (2014) Aerodynamics for Engineers, Sixth Edit. Pearson Education Limited, United Kingdom

18. Sharma G, Gupta PC, Kant S (2019) CFD Analysis of Two Dimensional Subsonic Flow Over S1223RTL Airfoil. Int J Eng Adv Technol 8:211-215

19. Altememe A, Myers OJ, Hall A (2019) Computational Fluid Dynamic Analysis of Flapping Wing of Micro Aerial Vehicle at Very Low Reynolds Numbers Turbulent Flow. Int J Aeronaut Aerosp Eng Res 1:68-75. https://doi.org/10.18689/ijae-1000110

20. Ananda GK, Sukumar PP, Selig MS (2015) Measured aerodynamic characteristics of wings at low Reynolds numbers. Aerosp Sci Technol 42:392-406. https://doi.org/10.1016/j.ast.2014.11.016

21. Launder BE, Spalding DB (1974) The Numerical Computation of Turbulent Flows. Comput Methods Appl Mech Eng 3:269-289. https://doi.org/10.1016/0045-7825(74)90029-2

22. Lawal MS, Fairweather M, Ingham DB et al (2010) Numerical study of emission characteristics of a jet flame in cross-flow. Combust Sci Technol 182:1491-1510.

https://doi.org/10.1080/00102202.2010.496379

23. Shih T-H, Liou WW, Shabbir A et al (1995) A new k- $\epsilon$ eddy viscosity model for high reynolds number turbulent flows. Comput Fluids 24:227-238. https://doi.org/10.1016/0045-7930(94)00032-T

24. Mahmud S, Mohamad W (2013) Introduction to ANSYS Workbench. 1-75 
25. ANSYS Inc (2013) ANSYS Fluent Theory Guide. 15317:724-746

26. Hamza A (2020) Flight Performance of ICHOKU-18 Unmanned Aerial Vehicle. Air Force Insitute of Technology, Kaduna

27. Jemitola PO, Abbe GE (2018) Medium Altitude Long Endurance Surveillance Aircraft IU-18 Project Specification

28. Zhang C, Bounds CP, Foster L, Uddin M (2019) Turbulence Modeling Effects on the CFD Predictions of Flow over a Detailed Full-Scale Sedan Vehicle. Fluids 4:1-28.

https://doi.org/10.3390/fluids4030148

\section{Figures}

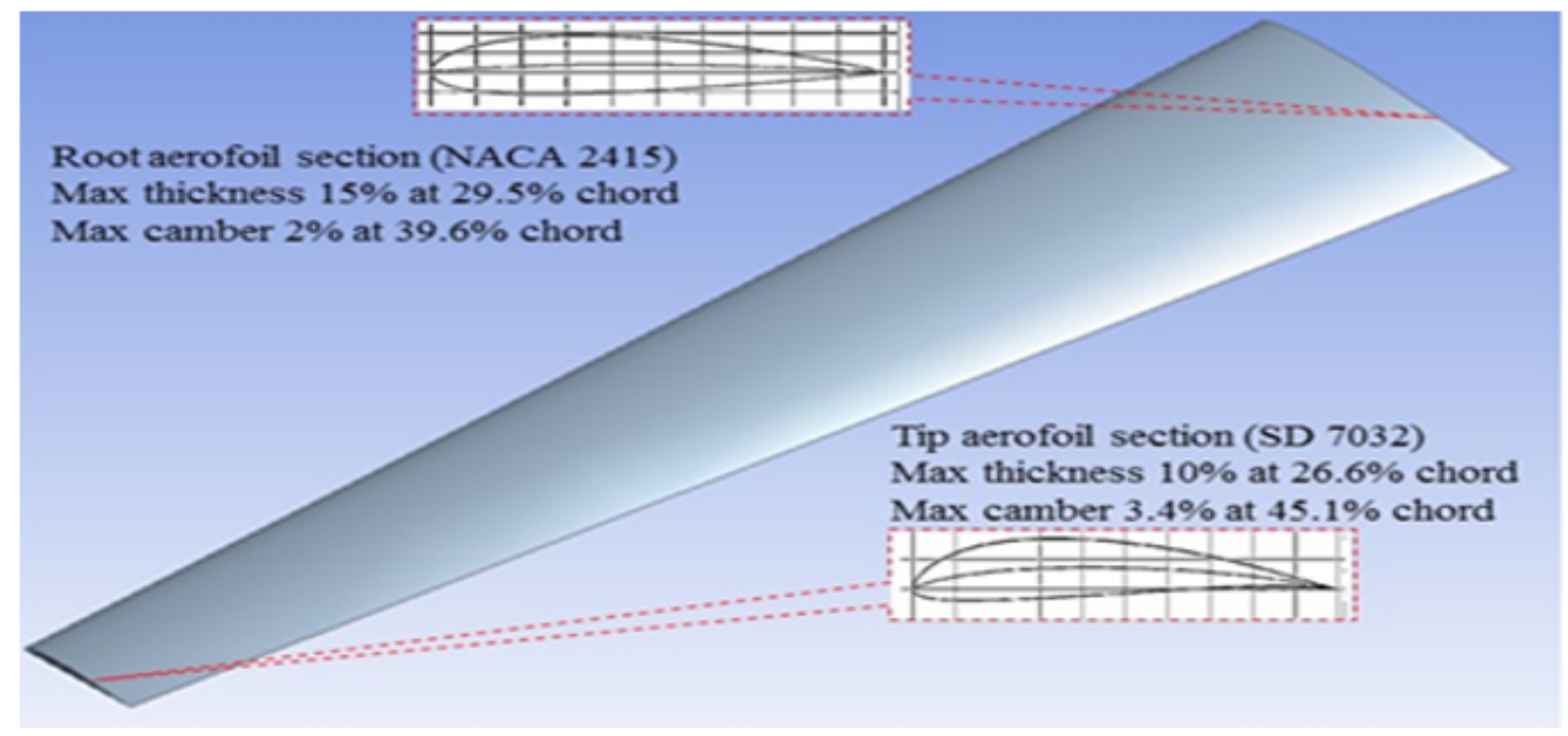

\section{Figure 1}

Wing CAD model produced. 


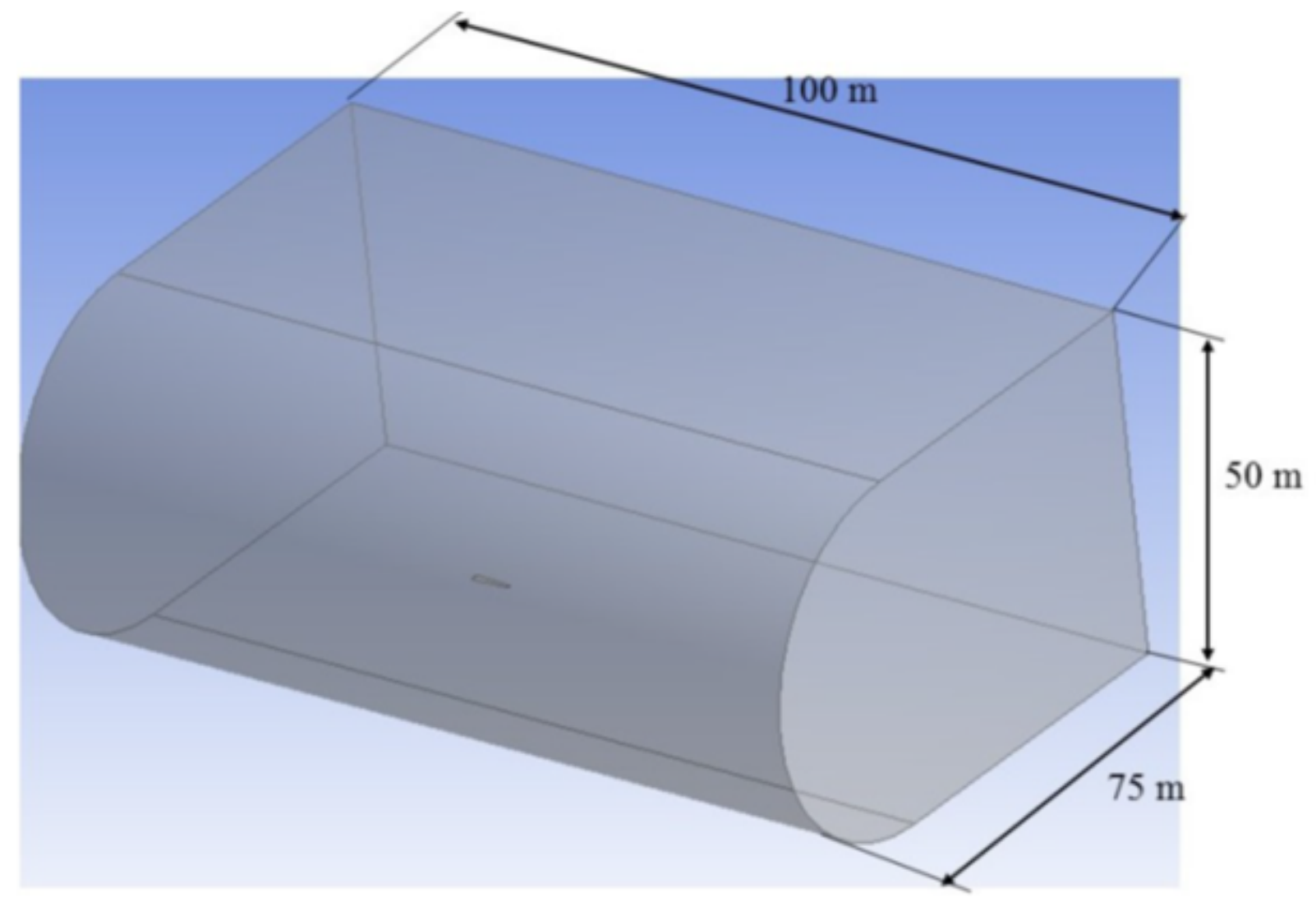

Figure 2

C-type CD designed. 


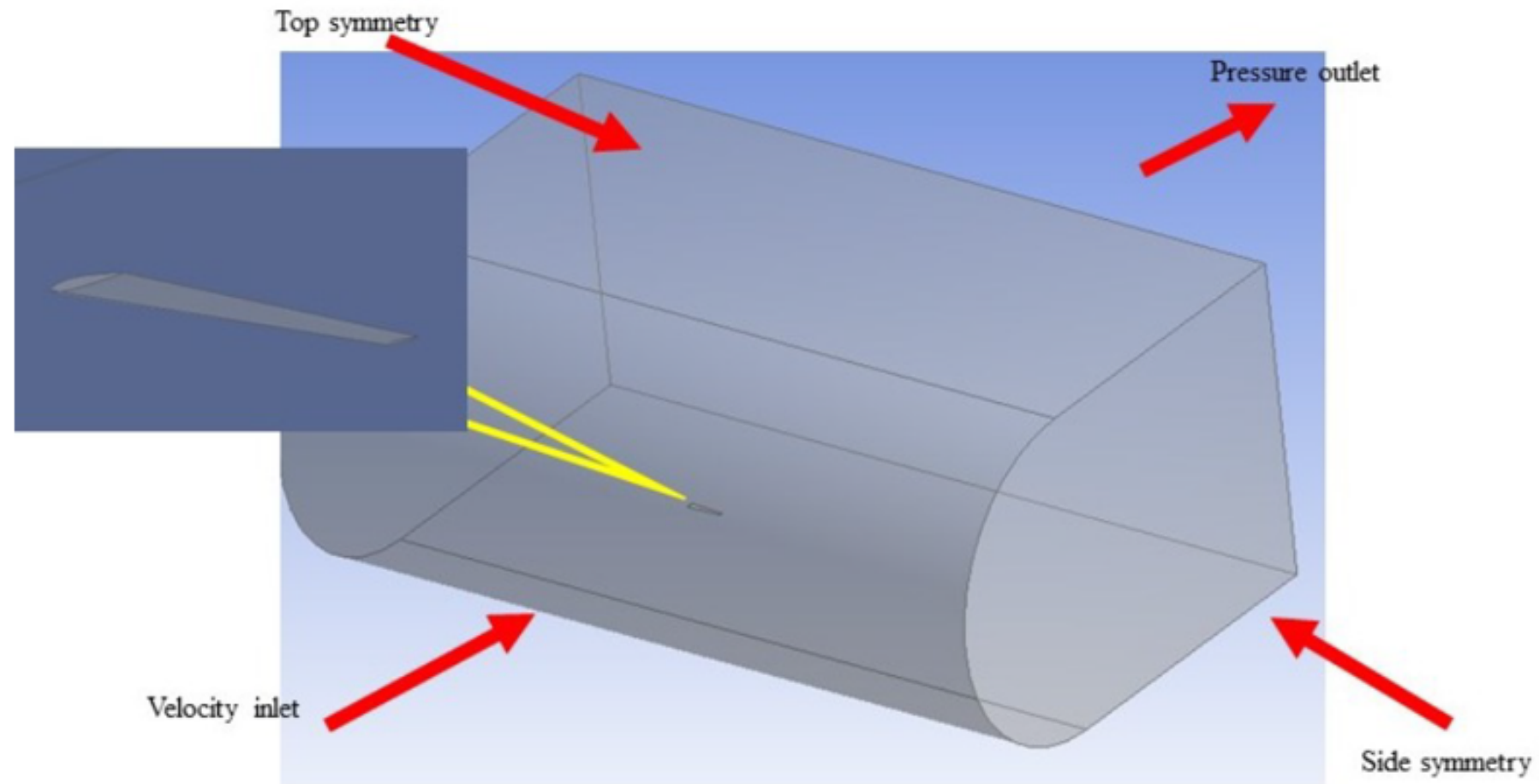

Figure 3

Boundaries conditions specified 


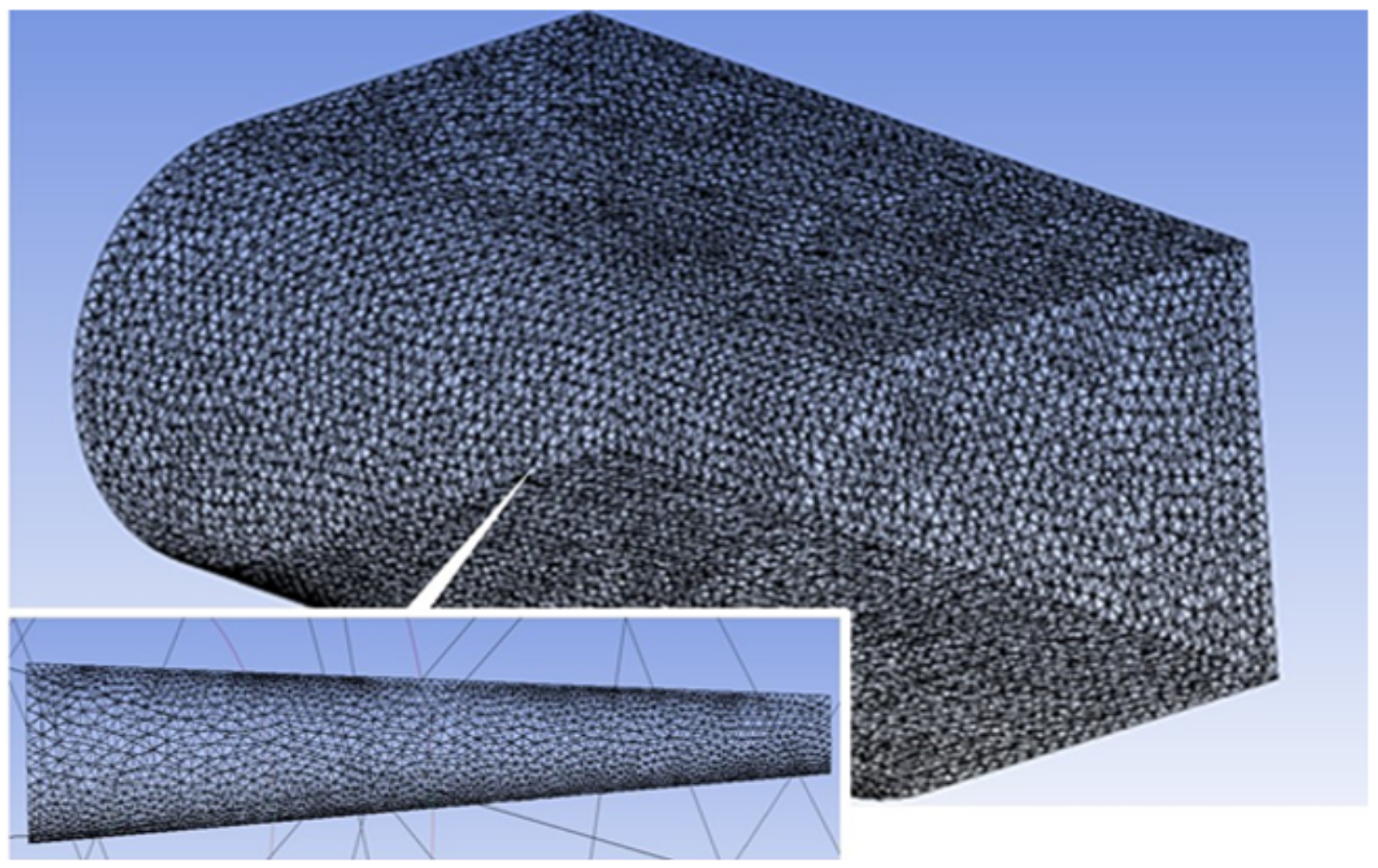

Figure 4

Meshed CD and UAV wing. 


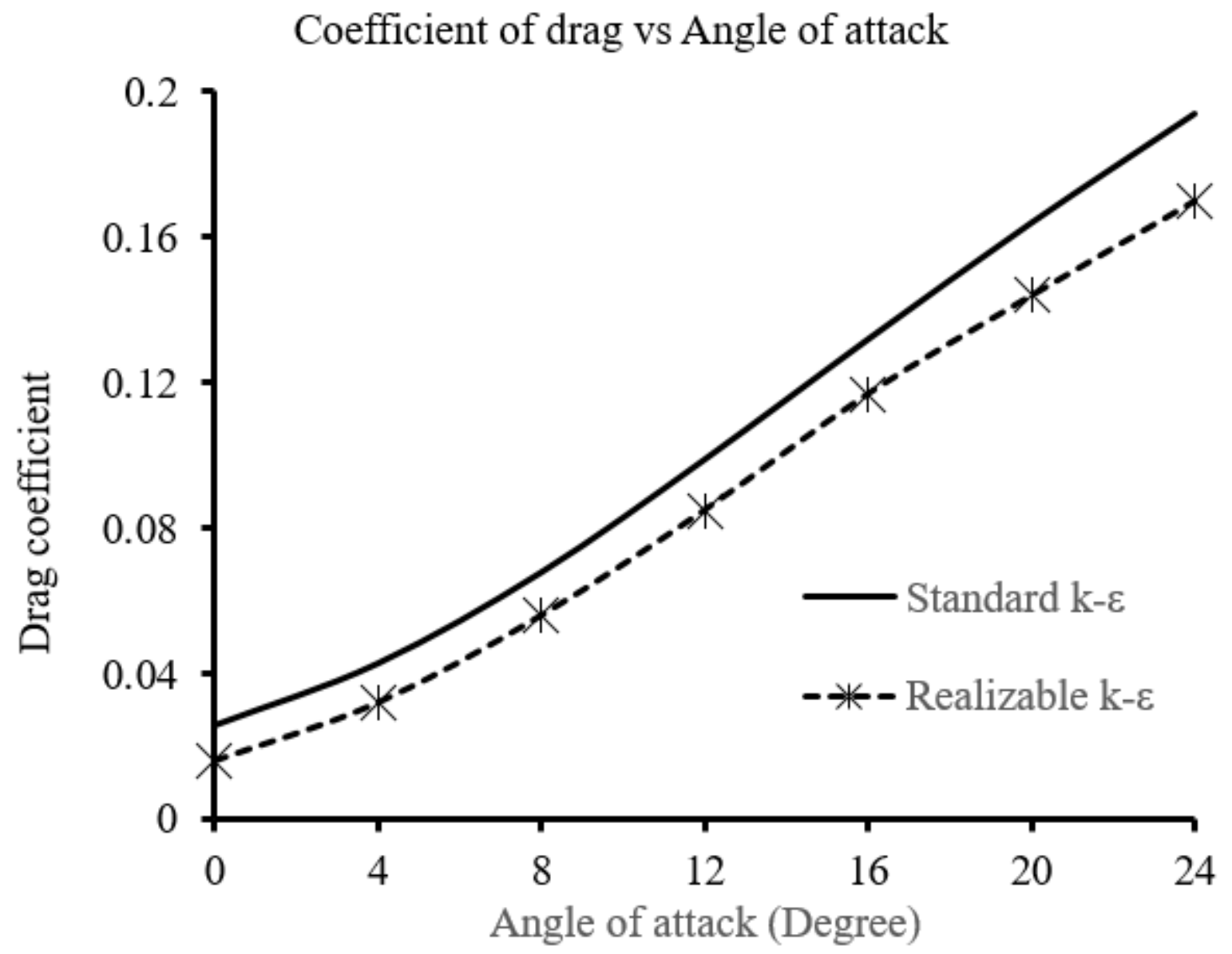

Figure 5

Curves of variation of against AoA. 


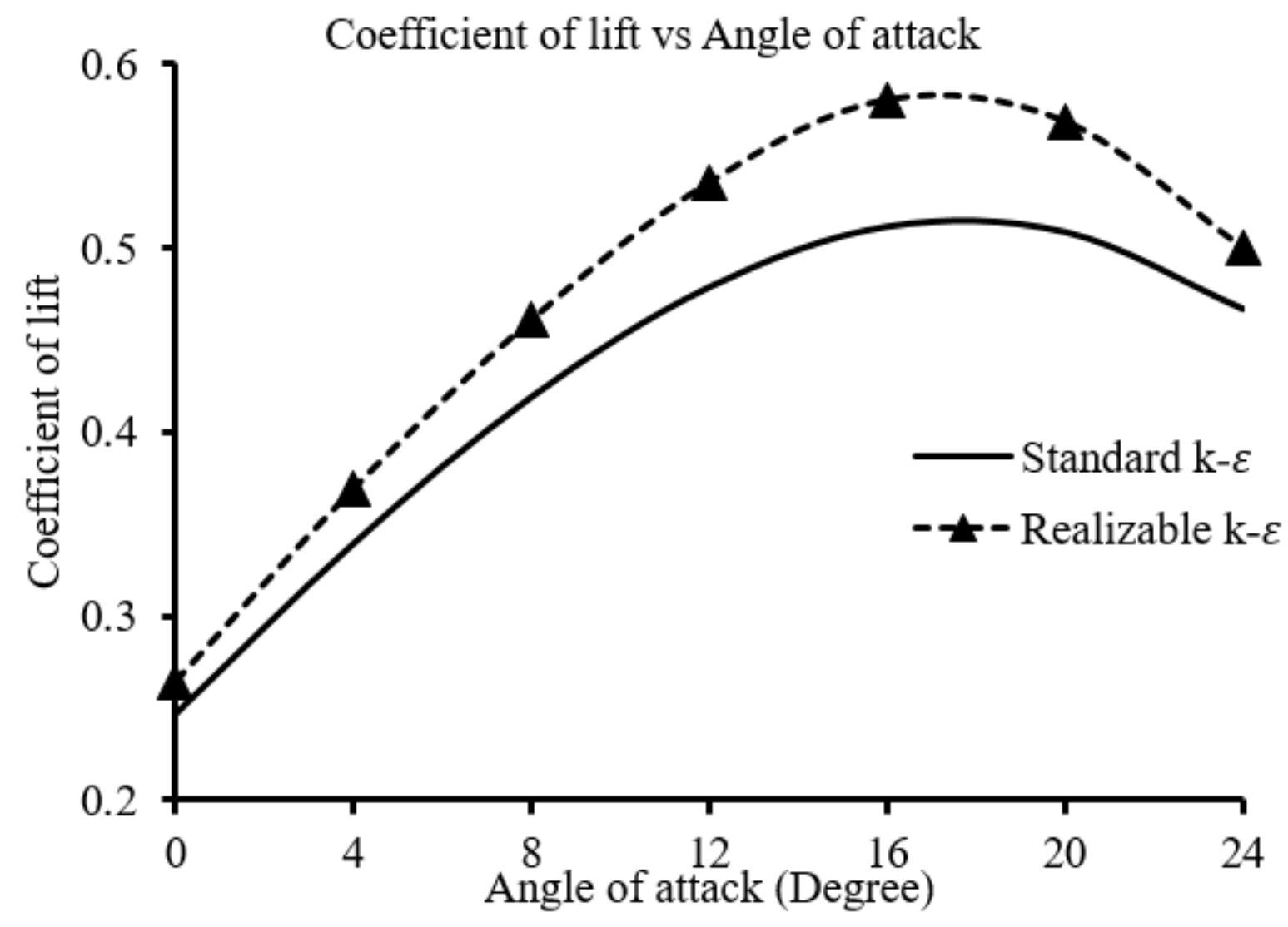

Figure 6

Curves of variation of lift coefficients against AoA. 


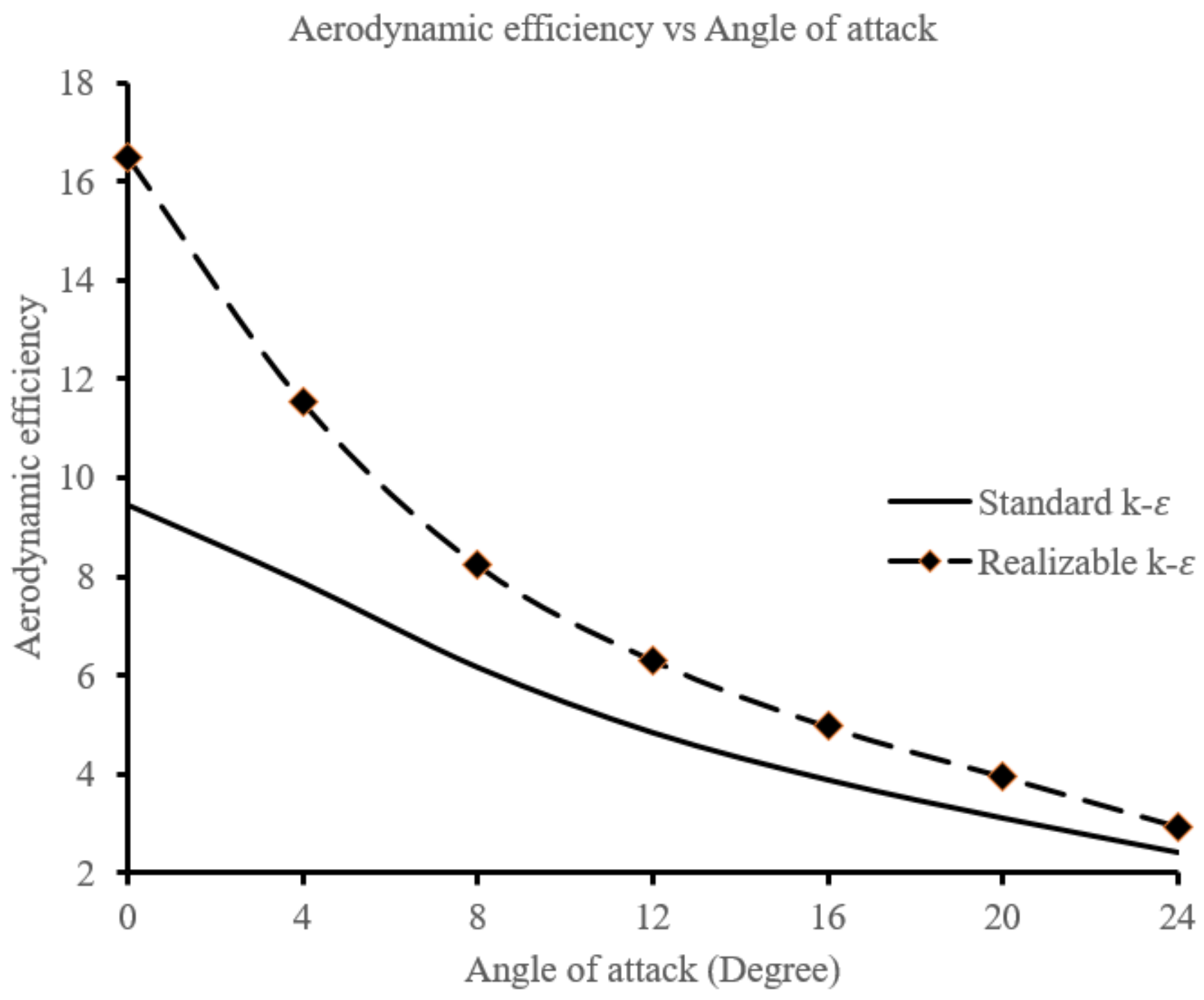

Figure 7

Curve of variation in aerodynamic efficiency against AoA. 